\title{
A Comparison of Resting Scapular Posture and the Davies Closed Kinetic Chain Upper Extremity Stability Test
}

\author{
John D Heick, PT, PhD, DPT, OCS, NCS, SCS ${ }^{1}$, Jenna Haggerty, PT, DPT ${ }^{1}$, Robert C Manske, PT, DPT, MPT, Med, SCS, \\ ATC, CSCS $^{2}$ \\ 1 Department of Physical Therapy and Athletic Training, Northern Arizona University, ${ }^{2}$ Department of Physical Therapy, Wichita State University \\ Keywords: upper extremity performance tests, movement system, kibler scapular classifications, davies closed kinetic chain upper extremity stability \\ test \\ https://doi.org/10.26603/001c.23425
}

\section{International Journal of Sports Physical Therapy}

Vol. 16, Issue 3, 2021

\begin{abstract}
BACKGROUND
In orthopaedic practice, it is well established that weak scapular stabilizers and an unstable scapula is related to shoulder dysfunction. Faulty scapular position has been linked to decreased scapular stability and is thought to be a result of weak or unbalanced timing in the recruitment of scapulothoracic dynamic stabilizing muscles. Kibler has described a four-type classification of scapulothoracic dysfunction. Functional performance testing is used to objectively measure activities that simulate various desired activities. The reliability of assessing the four static scapular positions may be important in diagnosing shoulder dysfunction. An understanding of the scapular position and its relationship to functional performance testing is needed.
\end{abstract}

\section{PURPOSE}

The purpose of this study was to determine if a static scapular test, the Kibler scapula classification, in healthy participants affects the ability to perform a closed chain functional test that involves the use of the scapula and the upper extremity, the Davies Closed Kinetic Chain Upper Extremity Stability Test (CKCUEST). A secondary analysis was performed to evaluate the reliability of a student physical therapist and an experienced physical therapist to identify scapular type by observation.

\section{STUDY DESIGN}

Multicenter, single session descriptive cohort

\section{METHODS}

Sixty-one healthy participants (33 males, 28 females; mean age 24.19 \pm 2.61 ) completed testing across two locations in one testing session. Blood pressure and heart rate as well as height and weight were measured for each participant. Participants were classified by visual observation of Kibler scapular classification. The average number of CKUEST touches, a normalized score, and a power score were calculated for each participant. Three trials were performed and participants were required to take a 45 -second rest break between each CKCUEST trial.

\section{RESULTS}

One way analysis of variance (ANOVA) showed statistically significant differences in Type I and Type IV Kibler scapula classification for the CKCUEST power score, however when an ANCOVA controlled for body mass index, there was no statistically significant

\footnotetext{
Corresponding author:

John D. Heick, PT, PhD, DPT

Northern Arizona University

PO Box 15105, Flagstaff, Arizona 86011

Telephone: 928-523-8394, fax: 928-523-9289

e-mail: John.Heick@nau.edu
} 
difference. A strong correlation $\mathrm{r}=.94$ was observed between student and experienced physical therapist in evaluating all four types of Kibler scapular classification.

\section{CONCLUSIONS}

Visually observed Kibler scapular position does not affect the ability to perform the Davies CKCUEST in healthy young adults. The ability to identify Kibler scapular position was reliable between student and experienced physical therapists. Additional studies are required to identify the usefulness of the Kibler scapular position classification.

\section{LEVEL OF EVIDENCE}

2b: Individual Cohort Study

\section{INTRODUCTION}

Alterations of the resting scapula position and dynamic scapular motion are frequently observed by sports physical therapists in patients with shoulder disorders from overuse injury in the overhead athlete. ${ }^{1-3}$ These alterations have been collectively classified by Kibler, ${ }^{4-7}$ three of which are thought to result in abnormal scapulohumeral rhythm and shoulder arthrokinematics and one that is considered normal scapular position. ${ }^{7}$ Abnormal scapulohumeral rhythm or dyskinesia can be considered either a cause or a result of shoulder disorders and adversely affect function. ${ }^{8}$ Evaluation of the Kibler classification can help in developing strategies to address shoulder dysfunction. During evaluation of the patient with shoulder dysfunction, scapular position should be examined statically and dynamically. ${ }^{9}$ The Kibler classification examination is a static scapular position test except for the last portion which involves observation of the position of the scapula in full shoulder flexion.

In terms of upper extremity performance, the Davies Closed Kinetic Chain Upper Extremity Stability test (CKCUEST) should be considered. The CKCUEST is a unique dynamic physical performance test because it assesses upper extremity function while most physical performance tests are related to the lower extremity. ${ }^{10-12}$ Most upper extremity sport activities involve closed kinetic chain activities. Closed kinetic chain upper extremity activities promote proprioception, joint stability, and high levels of activation of the scapular dynamic stabilizer muscles. ${ }^{13-17}$

The CKCUEST requires stability of the muscles that attach to the scapula, therefore, the CKCUEST may also be used as a scapular stability assessment. These scapular stabilizing muscles are active in both open and closed kinetic chain activities. ${ }^{17,18}$ The CKCUEST is a valid and reliable test that provides a measure of power and upper extremity stability. ${ }^{10,19}$ To perform the test, the participant is asked to assume a push-up position (men) or modified push-up position (females) and perform maximal speed alternating reaches to a tape target spaced 36 inches apart for $15 \mathrm{sec}-$ onds. The number of touches to the tape is recorded for three 15-second trials. Three potential scoring outcome measures can be calculated from the CKCUEST: average number of touches, a normalized score, and a power score. ${ }^{20-22}$

The primary purpose of this study was to determine if a static scapular test, the Kibler scapula classification, in healthy participants affects the ability to perform a closed chain functional test that involves the use of the scapula and the upper extremity, the Davies CKCUEST. A secondary analysis was performed to evaluate the reliability of the student physical therapist and experienced physical therapist to identify scapular type by observation. The hypotheses were that those with a Type IV or normal Kibler scapula classification would perform better on the Davies CKCUEST and that students and experienced therapists would have a moderate $(>0.60)$ inter rater reliability of identifying scapular type by observation.

\section{METHODS}

\section{PARTICIPANTS}

Sixty-three healthy participants were recruited across two locations. Participants were recruited from two public university campuses by flyer and word of mouth from Wichita State University and Northern Arizona University. The local institutional review boards approved the study. All participants were informed of the benefits and risks of the study before signing an institutionally approved informed consent document to participate. Participants were required to be between 18 to 40 years old to participate and to be able to speak English. Exclusion criteria for the study included: currently pregnant, current bout of shoulder or upper body pain, diagnosis of any shoulder condition in the past year, diagnosed hypertension or respiratory distress. All participants were asked about each of these conditions to ensure appropriate ability to participate in this study.

\section{INSTRUMENTATION}

\section{KIBLER SCAPULAR CLASSIFICATIONS}

Scapular classifications were described first by Kibler. ${ }^{7}$ Type I represents abnormal scapular control about a horizontal axis. Type II represents abnormal scapular control about a vertical axis. Type III represents excessive upward movement and abnormal control around a sagittal axis. Type IV is normal with bilaterally symmetric scapula. Multiple authors have investigated Kibler scapular classifications in the resting position that is with the participant standing in their normal posture with both arms at their sides therefore this was the case for this study. ${ }^{4,6,23,24}$ In the current study, Kibler scapular classifications were identified by visual observation.

\section{DAVIES CLOSED KINETIC CHAIN UPPER EXTREMITY STABILITY TEST}

To perform the CKCUEST, the participant is asked to assume 
Table 1: Demographic Characteristics of the Participants $(\mathrm{N}=61)$ of the Current Study

\begin{tabular}{|c|c|c|c|c|}
\hline Demographic Characteristic & No. (\%) & Weight (kg) a & Height $(m)^{a}$ & $\operatorname{Age}(y)^{a}$ \\
\hline \multicolumn{5}{|l|}{ Sex } \\
\hline Male & $33(54.1)$ & $77.53(8.47)$ & $1.80(.05)$ & $24.24(1.97)$ \\
\hline Female & $28(45.9)$ & $24.14(3.25)$ & $1.66(.07)$ & $24.14(3.25)$ \\
\hline Kibler type I & $20(33)$ & 68.65 (9.78) & $1.66(.07)$ & $23.90(1.21)$ \\
\hline Kibler type II & $11(18)$ & $65.56(14.12)$ & $1.69(.11)$ & $24(3.00)$ \\
\hline Kibler type IV & $30(49)$ & $75.91(9.75)$ & $1.76(.08)$ & $24.47(3.15)$ \\
\hline
\end{tabular}

a Weight, height and age are reported as mean (SD).

a push-up position and perform maximal speed alternating reaches to a tape target spaced 36 inches apart for $15 \mathrm{sec}-$ onds. The number of touches to the tape is recorded for three 15 -second trials. ${ }^{12}$ Some authors modify the push-up position for females by having the female in a kneeling position with knee support ${ }^{20,22,25}$ whereas others have concluded that the distance of the placement of the tape that is being reached to corresponds to anthropometric characteristics and maturational stages of the participant ${ }^{11,22}$ potentially putting a participant with a narrower build at a disadvantage. Authors have also modified the CKCUEST by performing only one trial ${ }^{11}$ or two trials ${ }^{26}$ as opposed to three trials. ${ }^{10,21,22}$ In the current study, female participants were offered a choice of the modified position or the standard push-up position and performed all three trials of the CKCST. Authors have suggested that CKCUEST scores can provide three potential scoring outcome measures from the CKCUEST: average number of touches, a normalized score, and a power score. ${ }^{20,22}$ The CKCUEST normalized score is obtained by dividing the number of touches by the height of the participant. ${ }^{20,22}$ The CKCUEST power score is obtained by multiplying the average touches by $68 \%$ of the participant's body weight $(\mathrm{kg})$ divided by $15 .^{20,22}$

\section{PROCEDURES}

Healthy participants aged 18 to 40 years old were recruited via flyers and advertising around the community. The order of procedures was the same for all participants. Each participant was weighed and height was measured followed by blood pressure and pulse rate being measured by a student physical therapist at both locations. Blood pressure was measured following the Frese et $\mathrm{al}^{27}$ guidelines and $\mathrm{ab}$ normal blood pressure or pulse rate excluded the participant from participation. A student physical therapist asked each participant to change into clothes to allow for visual observation of bilateral Kibler scapular positions with the participants limbs resting by the participant's side and then the student physical therapist recorded the result. The student physical therapist then invited the faculty member into the room to evaluate bilateral Kibler scapular positions and record their results. One student and one experienced physical therapist evaluated Kibler position in each setting.

The student physical therapist then reviewed the Davies CKCUEST procedure with the participant. For all partici- pants, female and male, two pieces of tape located 36 inches or 91.4 centimeters apart were placed on the floor. The participant was asked to assume a push up position and this became the starting position for the participant. Initially, the Davies CKCUEST starting position was performed with the hands spread apart to be touching the tape. Recently authors have suggested that placement of the hands in the starting position should correspond to the anthropometric characteristics of the participant. ${ }^{20,28}$ The participant was asked to move as quickly as possible alternating their reach to each piece of tape. A warmup trial was done to familiarize each participant before the three trials of the test. Between each trial, the participant was provided a 45 -second rest as per protocol. ${ }^{20,22,25}$ The average of the three trials was used as the test score for each participant.

\section{STATISTICAL METHODS}

Sample size calculation was based on having $80 \%$ power for the primary outcome of scapular posture and performance on the Davies CKCUEST. Data were analyzed using SPSS v24 (IBM Inc, Armonk, NY). A p-value of less than .05 was considered statistically significant. A one-way analysis of variance (ANOVA) was used to examine the effect of Kibler scapular type to the CKCST and an analysis of co-variance (ANCOVA) using body mass index (BMI) as a control. All assumptions were met to run the ANCOVA to evaluate the covariate. A prospective design was used to evaluate the reliability via Pearson Correlation Coefficients between student physical therapists and experienced physical therapists in identifying Kibler scapular type as correct or incorrect assessment.

\section{RESULTS}

The demographic characteristics of the 61 participants who completed testing (33 males, 28 females; mean age $24.19 \pm 2.61$ ) are provided in Table 1 . The most common Kibler scapular type was that of a type IV normal scapular position seen in 30 (49\%) participants, followed by Type I seen in 20 (33\%) of the participants. Two participants were excluded from participation in the study secondary to unidentified high blood pressure that did not resolve after resting five minutes. 
Table 2: Kibler Scapula Classification (type) with Performance of Davies Closed Kinetic Chain Upper Extremity Stability Test with Mean and Standard Deviations for Normalized Score, Age, Average Touches, and Power Score $(\mathrm{N}=61)$

\begin{tabular}{cccc}
\hline Kibler Type & CKCUEST & Mean (SD) & $95 \% \mathrm{Cl}$ \\
\hline & Normalized score & $14.80(2.33)$ & $13.71,16.05$ \\
\multirow{2}{*}{1} & Age & $23.90(1.21)$ & $22.70,25.11$ \\
& Average touches & $26.24(3.66)$ & $24.37,28.37$ \\
& Power score & $81.55(19.06)$ & $75.95,92.37$ \\
\hline & Normalized score & $14.09(3.73)$ & $12.62,15.79$ \\
& Age & $24.00(3.00)$ & $22.38,25.63$ \\
& Average touches & $24.65(6.05)$ & $22.15,27.55$ \\
& Power score & $72.91(22.35)$ & $65.62,87.80$ \\
\hline & Normalized score & $14.83(2.26)$ & $13.77,15.71$ \\
& Age & $24.46(3.14)$ & $23.46,25.45$ \\
& Average touches & $26.83(4.21)$ & $25.02,28.31$ \\
\end{tabular}

CKCUEST $=$ Closed Kinetic Chain Upper Extremity Stability Test; SD= standard deviation; CI=confidence intervals The CKCUEST normalized score is obtained by dividing the number of touches by the height of the participant

The CKCUEST power score considers the weight of the participant by multiplying the average number of touches by $68 \%$ of the participant's weight in kilograms.

\section{KIBLER SCAPULAR CLASSIFICATION AND THE CKCUEST}

Table 2 provides the means and $95 \%$ confidence intervals (CIs) for the average number of touches, normalized and power score for the CKCUEST for all participants. Results of the one way ANOVA showed statistically significant differences between participants with Type I and Type IV Kibler scapular classification for the CKCUEST power score but no differences for the average number of touches or the normalized scores. Results of the ANCOVA, when controlled for the body mass index revealed no significant differences between participants with differing Kibler scapular classifications and the CKCUEST power score (Figures $\underline{1}, \underline{2}, \underline{3}$ ). Of note, females that chose the modified push-up position for the CKCUEST accounted for $5 \%$ of participants.

\section{RELIABILITY OF SCAPULAR CLASSIFICATION BETWEEN THE STUDENT PHYSICAL THERAPIST AND THE EXPERIENCED PHYSICAL THERAPIST}

Pearson Correlation Coefficients between the student physical therapist and the experienced physical therapist at both locations are presented in Table 3 . The inter rater reliability of student physical therapists and experienced physical therapist from both locations was excellent in evaluating the Kibler scapular classification (ICC $=.96$; 95\% CI, 0.92, 0.99).

\section{DISCUSSION}

The purpose of this study was to determine if the Kibler scapular classification affects the ability to perform the CKCUEST in healthy participants. The first hypothesis that those with a normal Kibler scapula classification would perform better on the Davies CKCUEST was rejected. The re-

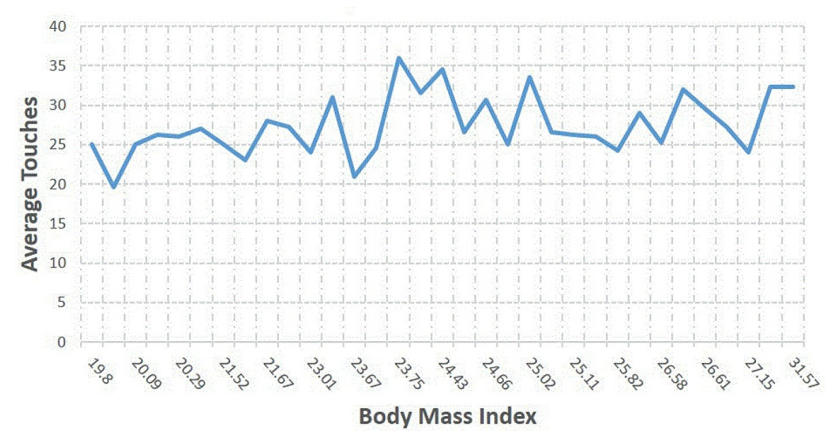

Figure 1: Average CKCUEST touches, males

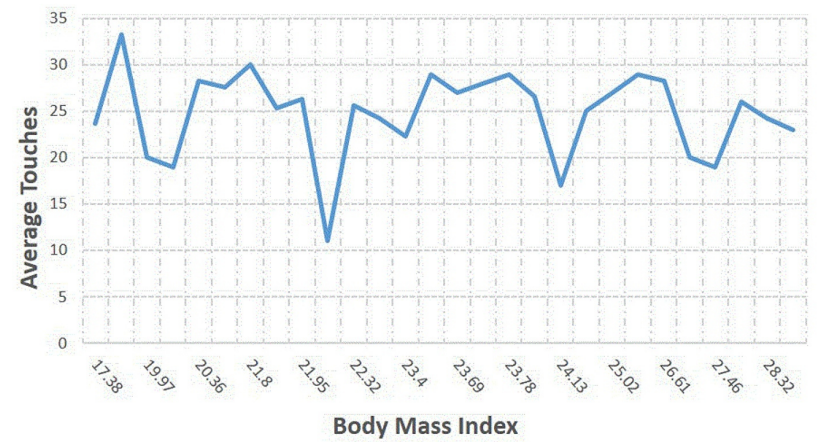

Figure 2: Average CKCUEST touches, females

sults from the current study show that Kibler scapula position does not affect the ability to perform the CKCUEST in healthy young adults. Identifying Kibler scapular position(s) has been clinically useful to describe static scapular positions but may not reflect functional scapular abilities. 
Table 3: Pearson Correlation Coefficients for the Kibler Scapula classification and Davies Upper Extremity Closed Kinetic Chain Upper Extremity Stability Test for both males and females at Both Locations (N=61)

\begin{tabular}{ccc}
\hline Trial & $\begin{array}{c}\text { Correlation between Kibler and CKCUEST at Northern } \\
\text { Arizona University }\end{array}$ & $\begin{array}{c}\text { Correlation between Kibler and CKCUEST at Wichita } \\
\text { State University }\end{array}$ \\
\hline 1 & $0.98(0.94,0.99)$ & $0.96(0.92,0.98)$ \\
\hline
\end{tabular}

95\% Confidence Intervals in parentheses

Deng et $\mathrm{a}^{23}$ investigated four different positions (at rest and end range of elevation in the sagittal, scapular and coronal planes) and found that $90.8 \%$ of patients with shoulder complaints had an abnormal resting scapular position when the patients' arms were by their side and at rest. Deng et $\mathrm{al}^{23}$ described this as scapular dyskinesia, which the authors of the current study would suggest as incorrect because dyskinesia as a clinical description implies upper extremity motion and instead abnormal resting position was found in a static position, which is not the true resting position of the scapula. Forty-nine percent of healthy participants in the current study had normal or type IV Kibler scapula classifications. None of the healthy participants with type I or type II had any symptoms.

Differences were only identified between those with Type I and Type IV Kibler scapular classification for the CKCUEST power score. Additionally, when controlled for body mass index no significant differences were present between participants with varied Kibler scapular classification types and the CKCUEST power score. Perhaps the lack of association is because it is a comparison of a visual observation of a static scapula position being compared to a dynamic stabilization test of the scapula stabilizers. Another potential reason may be that those with Kibler Type I scapular dyskinesis may represent a "normal asymmetry" in a healthy population. Future studies should include a larger sample size that includes subjects with a variety of Kibler scapular positions.

The interrater reliability of a second year student physical therapist at two separate locations to identify Kibler scapula types was excellent when compared to the experienced physical therapist. This is in contrast to authors who have noted low interrater reliability $(\mathrm{k}=0.084)$ in evaluating Kibler scapula classifications of uninjured professional baseball players. ${ }^{6}$ McClure et $\mathrm{al}^{29}$ investigated 142 uninjured athletes and found satisfactory reliability $(\mathrm{k}=0.48$ to 0.68 ) with percent agreement ranging between $75 \%$ to $82 \%$ but they evaluated both a static limbs position and asked participants to go through flexion and coronal plane abduction using a 3 to $5 \mathrm{lb}$ barbell. The current study approach did not include barbells to influence visual observation of Kibler classification. In terms of side-to side comparison, authors have found low interrater reliability for classifying the Kibler scapular position ( $\mathrm{k}=0.264$ for left, $\mathrm{k}=0.157$ for right). 6,30 The ability of a first year or novice student and an experienced physical therapist to reliably agree on the scapular posture by observation examination is presently unknown. Authors have also reported a limitation of interpretation of scapular position with video analysis. ${ }^{6,30} \mathrm{Mc}-$

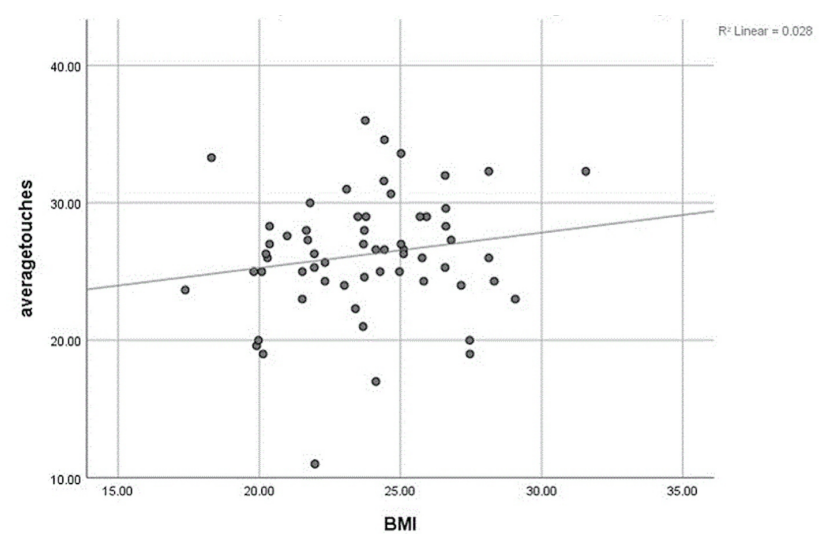

Figure 3: Scatter plot for correlation between average touches and body mass index (BMI

Clure et al also used video analysis for interpretation and reported coefficients ranging between 0.48 and 0.61 with percentage agreement ranging from $75 \%$ to $82 \%$ between examiners. ${ }^{29}$ Tate et al tried to validate the McClure et al study by using 66 of the same 142 participants used in the McClure et al study. ${ }^{31}$ Tate et $\mathrm{al}^{31}$ used three-dimensional electromagnetic kinematic testing and noted less scapular upward rotation or Type III scapula in the participants that McClure et $\mathrm{al}^{29}$ investigated and classified. Thus, visual observation of scapular position overestimated the three-dimensional electromagnetic kinematic testing or more simply stated what they saw did not correspond to precise measurements of scapular motion. Uhl et $\mathrm{al}^{32}$ categorized symptomatic and asymptomatic athletes into a yes/no method in which those with abnormal scapular dyskinesia were labeled as having dyskinesia and were not specifically categorized by scapular type. They found a sensitivity of $76 \%$ and positive predictive value of $74 \%$ using the yes/no method. ${ }^{32}$ Interestingly, they also noted that testing symptomatic patients showed a higher frequency of multipleplane scapular asymmetries. ${ }^{32}$ Deng et $\mathrm{al}^{23}$ investigated the Kibler classification system in four different positions but concluded that the resting position of the scapula was the best position to assess in patients with shoulder dysfunction. In the current study, this static resting position of the scapula was reliable between student and experienced clinician but was not associated with CKCUEST performance.

Authors investigating the CKCUEST have suggested that the test has excellent reliability for adolescents, ${ }^{22}$ for physically active adults, ${ }^{21}$ sedentary adults, and sedentary adults diagnosed with shoulder impingement syndrome, ${ }^{21}$ for Di- 
vision I college athletes, ${ }^{11,26}$ collegiate basketball athletes, ${ }^{33}$ for handball athletes, ${ }^{34}$ and collegiate baseball athletes. ${ }^{10}$ The majority of these studies have examined test-retest reliability and three of these studies investigated and reported the average number of touches, normalized score, and power score. ${ }^{20,22,28}$

Use of the power score and normalized score requires further investigation. Goldbeck and Davies ${ }^{12}$ first described the power score using $68 \%$ of the participant's weight in kilograms based on Dempster's work. ${ }^{35}$ The power score considers the weight of the participant by multiplying the average number of touches by $68 \%$ of the participant's weight in kilograms. Tucci et $\mathrm{al}^{20,21}$ used the power score and report that $68 \%$ of the participant's body weight in kilograms corresponds to the weight of the arms, head and trunk. Dempster ${ }^{35}$ is often cited as the classic paper for considering the mass of body segments but used adult male cadavers between 52 to 83 years of age to derive the $68 \%$. Recently, authors have suggested that using older male cadavers do not correspond to younger adults or appreciate sex differences. ${ }^{36,37}$ Authors have separated body segment mass by sex and suggest that the mass of the head, trunk, upper arms, forearms, and hands equal $60.28 \%$ for males and $58.23 \%$ for females of the total mass of the body. ${ }^{37,38}$ Virmavirta et $\mathrm{al}^{36}$ concluded that inter-individual differences among athlete groups may be large and that the selection of segment body mass for analysis is not clear. Virmavirta et $\mathrm{al}^{36}$ suggest using a precise body segment model specifically for athletes. For example, gymnasts have different segmental body mass compared to throwers or hockey players. Considering that the use of the power score may not be specific enough for athletes, the authors of the current study controlled for BMI for the average number of touches of the CKCUEST and for the power and normalized score and found no statistically significant relationship to Kibler scapular position (Figure 3). The authors of the current study suggest that the use of a power score of $68 \%$ should not be used for athletes or for females based on the variability found in the literature on segmental body mass for younger athletes. Instead, a power score of $60.28 \%$ should be used for males and $58.23 \%$ for females to be precise. This approach should be adopted until sport specific normative data for body segment mass are gathered in a large population of athletes.

The normalized score considers the height of the participant by dividing the number of touches by the height of the participant and has also been questioned. Authors have suggested that the height of the participant, the maturational stage, or anthropometric measurements such as shoulder width place for those with a narrower build creates a performance disadvantage for the CKCUEST. ${ }^{11,28}$ Callaway et $\mathrm{al}^{28}$ suggest that the original CKCUEST starting position of placement of the hands 36 inches apart is not justified. ${ }^{12}$ Callaway et $\mathrm{al}^{28}$ suggest that using 36 inches as the starting position is inappropriate for both sexes as taller participants will have a greater advantage over shorter participants which leads them to suggest a different starting position appropriate to the height of the participant. ${ }^{28}$ Callaway et $\mathrm{al}^{28}$ investigated four different starting positions (36 inches, shoulder breadth, acromial distance, and 50\% of the participant's height) and reported that the starting position at $50 \%$ of the participant's height resulted in the smallest standard error of measurement and minimal detectable change of all three positions. Callaway et $\mathrm{al}^{28}$ only investigated males in their study so it is unknown if performance improves in females using this starting position. Taylor et al $^{11}$ used shoulder width as the starting position for male and female collegiate athletes as an attempt to adjust for body-size heterogeneity of the athlete. In the current study, the authors offered the participant the choice of starting position to correspond to the shoulder width of the participant. Regarding the normalized score, the authors of the current study suggest more evidence is needed on the use of the normalized score. Specifically, it is important to have normative data on how height affects the starting position and performance of the CKCUEST across all types of athletes. The authors of the current study suggest that since the original paper on the CKCUEST investigated this test as an upper extremity performance test of endurance, three trials of the test should be performed and performance of fewer than three trials alters the purpose of the original test. Maintaining a standardized approach of three trials should be used in the clinic as the original intent of the CKCUEST is related to endurance.

The authors of the current study did exclude two participants secondary to unidentified high blood pressure that precluded them from participation in exercise. The authors of the current study suggest that future studies investigating the CKCUEST should take blood pressure measurements on all participants especially since upper extremity exercise increases systolic blood pressure much more so than lower extremity exercise. ${ }^{39,40}$ As a precaution, any patient that is unknown to a clinician that performs upper extremity exercise should have their blood pressure measured by their therapist. For example, a per diem physical therapist that is covering a holiday that does not know any of the patients should consider taking blood pressure measurements on unfamiliar patients due to the increase in systolic blood pressure during upper extremity specific exercise.

The authors of the current study identify that experimental studies have limitations. One limitation was that the order of testing was standardized (visual observation of Kibler classification followed by three trials of the CKCUEST) and therefore may not mimic the clinical setting. Another limitation is that the study was done at two separate locations involving healthy participants that self-reported if they had a shoulder injury over the past year or current shoulder pain. As the current study used only healthy participants, findings should be extrapolated with caution to those following rehabilitation for upper extremity injury. It is unknown if the participants had any actual previous shoulder injuries or other musculoskeletal conditions that were not identified.

\section{CONCLUSIONS}

Sports physical therapists commonly evaluate patients with upper extremity injury or conditions and observe abnormal scapular position in the affected extremity. In the current study, resting Kibler scapular position did not affect the ability to perform the Davies CKCUEST in healthy young adults. However, the ability to identify Kibler scapular posi- 
tions was reliable between second year students and experienced physical therapists. The authors suggest future studies should investigate varied starting positions and the use of the power and normalized scores for the CKCUEST.

\section{CONFLICTS OF INTEREST}

The authors do not have any conflict of interest to report.

Submitted: June 22, 2020 CDT, Accepted: December 15, 2020 CDT 


\section{REFERENCES}

1. Myers JB, Laudner KG, Pasquale MR, Bradley JP, Lephart SM. Scapular position and orientation in throwing athletes. Am J Sports Med. 2005;33(2):263-271. doi:10.1177/0363546504268138

2. Laudner KG, Stanek JM, Meister K. Differences in scapular upward rotation between baseball pitchers and position players. Am J Sports Med. 2007;35(12):2091-2095. doi:10.1177/03635465073050 98

3. Oyama S, Myers JB, Wassinger CA, Ricci RD, Lephart SM. Asymmetric resting scapular posture in healthy overhead athletes. J Athl Train.

2008;43(6):565-570. doi:10.4085/1062-6050-43.6.565

4. Kibler WB, Sciascia A. Current concepts: scapular dyskinesis. Br J Sports Med. 2010;44(5):300-305. doi:1 $\underline{0.1136 / \text { bjsm.2009.058834 }}$

5. Kibler WB, Kuhn JE, Wilk K, et al. The disabled throwing shoulder: spectrum of pathology-10-year update. Arthroscopy. 2013;29(1):141-161 e126. doi:1 0.1016/j.arthro.2012.10.009

6. Ellenbecker TS, Kibler WB, Bailie DS, Caplinger R, Davies GJ, Riemann BL. Reliability of scapular classification in examination of professional baseball players. Clin Orthop Relat Res. 2012;470(6):1540-1544. doi:10.1007/s11999-011-221 $\underline{6-0}$

7. Kibler WB. The role of the scapula in athletic shoulder function. Am J Sports Med. 1998;26(2):325-337. doi:10.1177/03635465980260022 $\underline{801}$

8. McQuade KJ, Borstad J, de Oliveira AS. Critical and Theoretical Perspective on Scapular Stabilization: What Does It Really Mean, and Are We on the Right Track? Phys Ther. 2016;96(8):1162-1169. doi:10.2522/ ptj.20140230

9. Wright AA, Wassinger CA, Frank M, Michener LA, Hegedus EJ. Diagnostic accuracy of scapular physical examination tests for shoulder disorders: a systematic review. Br J Sports Med. 2013;47(14):886-892. doi:10.1 136/bjsports-2012-091573

10. Roush JR, Kitamura J, Waits MC. Reference Values for the Closed Kinetic Chain Upper Extremity Stability Test (CKCUEST) for Collegiate Baseball Players. N Am J Sports Phys Ther. 2007;2(3):159-163.
11. Taylor JB, Wright AA, Smoliga JM, DePew JT, Hegedus EJ. Upper-Extremity Physical-Performance Tests in College Athletes. J Sport Rehabil. 2016;25(2):146-154. doi:10.1123/jsr.2014-0296

12. Goldbeck TG, Davies GJ. Test-retest of the Closed Kinetic Chain Upper Extremity Stability Test: a clinical field test. J Sport Rehabil. 2000;9:35-45.

13. Tucker WS, Armstrong CW, Gribble PA, Timmons MK, Yeasting RA. Scapular muscle activity in overhead athletes with symptoms of secondary shoulder impingement during closed chain exercises. Arch Phys Med Rehabil. 2010;91(4):550-556. doi:10.10 16/j.apmr.2009.12.021

14. Tucker WS, Bruenger AJ, Doster CM, Hoffmeyer DR. Scapular muscle activity in overhead and nonoverhead athletes during closed chain exercises. Clin J Sport Med. 2011;21(5):405-410. doi:10.1097/IS M.0b013e31822179e8

15. Prokopy MP, Ingersoll CD, Nordenschild E, Katch FI, Gaesser GA, Weltman A. Closed-kinetic chain upper-body training improves throwing performance of NCAA Division I softball players. J Strength Cond Res. 2008;22(6):1790-1798. doi:10.1519/JSC.0b013e31 $8185 f 637$

16. Kang MH, Oh JS, Jang JH. Differences in muscle activities of the infraspinatus and posterior deltoid during shoulder external rotation in open kinetic chain and closed kinetic chain exercises. J Phys Ther Sci. 2014;26(6):895-897. doi:10.1589/jpts.26.895

17. Rogol IM, Ernst G, Perrin DH. Open and closed kinetic chain exercises improve shoulder joint reposition sense equally in healthy subjects. $J$ Athl Train. 1998;33(4):315-318.

18. Uhl TL, Carver TJ, Mattacola CG, Mair SD, Nitz AJ. Shoulder musculature activation during upper extremity weight-bearing exercise. J Orthop Sports Phys Ther. 2003;33(3):109-117. doi:10.2519/jospt.200 3.33.3.109

19. Lee DR, Kim LJ. Reliability and validity of the closed kinetic chain upper extremity stability test. $J$ Phys Ther Sci. 2015;27(4):1071-1073. doi:10.1589/jpt s.27.1071

20. Tucci HT, Felicio LR, McQuade KJ, BevilaquaGrossi D, Camarini PM, Oliveira AS. Biomechanical Analysis of the Closed Kinetic Chain Upper-Extremity Stability Test. J Sport Rehabil. 2017;26(1):42-50. doi:1 0.1123/jsr.2015-0071 
21. Tucci HT, Martins J, Sposito Gde C, Camarini PM, de Oliveira AS. Closed Kinetic Chain Upper Extremity Stability test (CKCUES test): a reliability study in persons with and without shoulder impingement syndrome. BMC Musculoskelet Disord. 2014;15:1. do i:10.1186/1471-2474-15-1

22. de Oliveira VM, Pitangui AC, Nascimento VY, da Silva HA, Dos Passos MH, de Araujo RC. Test-retest reliability of the Closed Kinetic Chain Upper Extremity Stability Test (CKCUEST) in adolescents: Reliability of CKCUEST in adolescents. Int J Sports Phys Ther. 2017;12(1):125-132.

23. Deng S, Chen K, Ma Y, Chen J, Huang M. The influence of test positions on clinical assessment for scapular dyskinesis. PM R. 2017;9(8):761-766. doi:1 $\underline{0.1016 / j . p m r j .2016 .11 .011}$

24. Wassinger CA, Williams DA, Milosavljevic S, Hegedus EJ. Clinical reliability and diagnostic accuracy of visual scapulohumeral movement evaluation in detecting patients with shoulder impairment. Int J Sports Phys Ther.

2015;10(4):456-463.

25. Schulte-Edelmann JA, Davies GJ, Kernozek TW, Gerberding ED. The effects of plyometric training of the posterior shoulder and elbow. J Strength Cond Res. 2005;19(1):129-134. doi:10.1519/13963.1

26. Pontillo M, Sennett BJ. Profile of upper extremity strength and function in division 1 collegiate athletes. Phys Ther Sport. 2020;44:8-13. doi:10.1016/ j.ptsp.2020.03.003

27. Frese EM, Fick A, Sadowsky HS. Blood pressure measurement guidelines for physical therapists. Cardiopulm Phys Ther J. 2011;22(2):5-12.

28. Callaway A, Peck J, Ellis S, Williams J. A randomised observational study of individualised variations in the start position of the closed-kinetic chain upper extremity stability test. Phys Ther Sport. 2020;41:16-22. doi:10.1016/i.ptsp.2019.10.007

29. McClure P, Tate AR, Kareha S, Irwin D, Zlupko E. A clinical method for identifying scapular dyskinesis, part 1: reliability. J Athl Train. 2009;44(2):160-164. do i:10.4085/1062-6050-44.2.160

30. Kibler WB, Uhl TL, Maddux JW, Brooks PV, Zeller B, McMullen J. Qualitative clinical evaluation of scapular dysfunction: a reliability study. J Shoulder Elbow Surg. 2002;11(6):550-556. doi:10.1067/mse.200 $\underline{2.126766}$
31. Tate AR, McClure P, Kareha S, Irwin D, Barbe MF. A clinical method for identifying scapular dyskinesis, part 2: validity. J Athl Train. 2009;44(2):165-173. doi:1 0.4085/1062-6050-44.2.165

32. Uhl TL, Kibler WB, Gecewich B, Tripp BL. Evaluation of clinical assessment methods for scapular dyskinesis. Arthroscopy. 2009;25(11):1240-1248. doi:10.1016/j.arthro.2009.0 $\underline{6.007}$

33. Hollstadt K, Boland M, Mulligan I. Test-retest reliability of the Closed Kinetic Chain Upper Extremity Stability Test (CKCUEST) in a modified test position in division I collegiate basketball players. Int J Sports Phys Ther. 2020;15(2):203-209.

34. Decleve P, Van Cant J, De Buck E, Van Doren J, Verkouille J, Cools AM. The self-assessment corner for shoulder strength: reliability, validity, and correlations with upper extremity physical performance tests. J Athl Train. 2020;55(4):350-358. d oi:10.4085/1062-6050-471-18

35. Dempster WT. Space Requirements of the Seated Operator. Geometrical, Kinematic, and Mechanical Aspects of the Body with Special Reference to the Limbs. Springfield, Ohio; 1955:55-159.

36. Virmavirta M, Isolehto J. Determining the location of the bodys center of mass for different groups of physically active people. J Biomech. 2014;47(8):1909-1913. doi:10.1016/j.jbiomech.2014.0 4.001

37. de Leva P. Adjustments to Zatsiorsky-Seluyanov's segment inertia parameters. J Biomech. 1996;29(9):1223-1230. doi:10.1016/0021-9290(95)001 78-6

38. Zatsiorsky VM. Inertial Properties of the Human Body. Kinetics of Human Motion. Champaign, Illinois: Human Kinetics; 2002.

39. Machado-Vidotti HG, Mendes RG, Simoes RP, Castello-Simoes V, Catai AM, Borghi-Silva A. Cardiac autonomic responses during upper versus lower limb resistance exercise in healthy elderly men. Braz J Phys Ther. 2014;18(1):9-18. doi:10.1590/s1413-355520120 05000140

40. Di Blasio A, Sablone A, Civino P, D’Angelo E, Gallina S, Ripari P. Combined leg and arm exercise: Blood pressure responses and ratings of perceived exertion at the same indirectly determined heart rate. J Sports Sci Med. 2009;8(3):401-409. 